\title{
GESTIONANDO LA DIVERSIDAD EN LAS ORGANIZACIONES A TRAVÉS DE LAS COMUNIDADES. INVESTIGACIÓN CON MÉTODOS MIXTOS
}

\author{
Gutiérrez-Ortega, M. ${ }^{1}$, Martín-Cilleros, M.V. ${ }^{2}$ y Hernández-Soto, R $^{1}$ \\ 1 Universidad Internacional de la Rioja, España.monica.gutierrez@unir.net, roberto.hernandez@unir.net \\ 2 Universidad de Salamanca, España. viquimc@usal.es
}

\begin{abstract}
Resumen. Introducción La gestión de la diversidad en las organizaciones conlleva conocer aquellos factores personales, sociales y culturales que pueden interceder. Las comunidades que surgen en las organizaciones son contextos que pueden ayudar a promover la inclusión. Objetivos; Métodos Se presenta un estudio mixto basado en un diseño exploratorio secuencial para estudiar las percepciones ante la inclusión en una organización con un fuerte sentimiento de comunidad. Resultados Los resultados obtenidos en las fases cuantitativa y cualitativa nos indican la existencia de una percepción común de la inclusión y unos altos valores en todas las categorías definidas. Conclusiones. Los valores, las prácticas, las políticas y la cultura de una organización se alinean con la inclusión cuando en la organización se promueve el desarrollo de la comunidad. Los métodos mixtos nos han permitido abordar un constructo complejo desde diferentes perspectivas. Los resultados obtenidos en la fase cualitativa han enriquecido y complementado los obtenidos en la fase cuantitativa.
\end{abstract}

Palabras clave: Diversidad; Inclusión; Comunidad; Método Mixto; Cualitativo

\section{MANAGING DIVERSITY IN ORGANIZATIONS ACROSS COMMUNITIES. MIXED METHODS RESEARCH}

\begin{abstract}
Introduction The management of diversity in organizations involves knowing those personal, social, and cultural factors that may intercede. The communities that arise in organizations are contexts that can help promote inclusion. Goals; Methods We present a mixed study based on a sequential exploratory design to study the perception of inclusion in an organization with a strong sense of community. Results The results obtained in the quantitative and qualitative phases point to the existence of a common perception of inclusion and high values in all defined categories. Conclusions. The organizational values, practices, policies, and culture are aligned with inclusion when community development is promoted within the organization. Mixed methods have allowed us to approach a complex construct from different perspectives. The results obtained in the qualitative phase have enriched and complemented those obtained in the quantitative phase.
\end{abstract}

Keywords: Diversity; Inclusion; Community; Mixed Method; Qualitative

\section{INTRODUCCIÓN}

La diversidad en las organizaciones conlleva entendimiento y gestionarla va más allá de la simple tolerancia, implica la comprensión y el reconocimiento de cada individuo en una organización como único y diferente (Cox y Cox, 2001; Schein, 2004). Las diferencias pueden ser por razón de la raza, etnia, estatus socioeconómico, género, edad, capacidad o discapacidad, religión, creencias políticas, orientación sexual o ideología (Cox y Cox, 2001). 
No se debe perder de vista en las organizaciones que la diversidad puede tener un lado no tan positivo, ya que las idiosincrasias individuales pueden ser obstáculos para el desarrollo exitoso de una cultura inclusiva y variada.

Las organizaciones deben tener en cuenta todos aquellos factores personales, sociales y culturales que pueden interceder a la hora de implementar políticas inclusivas. Hay que tener presente que lo que hace unos años era "lógico" o "normal" poco a poco se va reinventando. $\mathrm{Y}$, en estos procesos de cambio, es dónde los nuevos significados y valores, que la inclusión lleva asociados, deben ir interpretándose e integrándose en el quehacer organizativo. Los nuevos significados se deberán ir negociando entre todas las personas que forman parte de la organización (Azorin \& Ainscow, 2018).

El sentimiento de comunidad que se puede dar en algunas organizaciones puede facilitar que sus miembros tengan un escenario que les permita expresar lo son y lo que quieren ser (Amor et al., 2019). Un escenario que les permita investigar sobre lo que hacen en el día a día y lo que les gustaría hacer para mejorar. De este modo, podrán, a la vez que siguen las dinámicas del día, ir adaptándose a los cambios que se producen tanto en la organización, como en la comunidad o en la sociedad (Hiebert, Gallimore \& Stigler, 2002; Ainscow \& Sandill, 2010).

El estudio exploratorio que se presenta tiene como objetivo examinar las siguientes preguntas:

- ¿En una organización con un fuerte sentimiento de comunidad hay percepciones compartidas asociadas a la inclusión?

- ¿La existencia de una comunidad hace que las percepciones sean comunes independientemente del rol, la edad o el sexo?

- ¿Una metodología de investigación mixta ayudará a profundizar en un constructo complejo como es el de la inclusión?

\section{METODOLOGÍA}

Por la complejidad del constructo a evaluar se ha diseñado una investigación que combina de enfoques cualitativos y cuantitativos (Creswell, 2014; Creswell et al., 2004; Tashakkori \& Teddlie, 2003). Se ha optado por un diseño exploratorio secuencial donde el método cuantitativo apoyará al método cualitativo que es el que marcará la investigación (Greene \& 
Caracelli, 2003). Por ello, la investigación se iniciará con la obtención de datos cualitativos para explorar la inclusión en la entidad seleccionada, y, posteriormente se llevará a cabo una etapa cuantitativa de la cual se extraerán resultados que se compararán e integrarán en la interpretación del análisis.

En la investigación cualitativa se realizaron observaciones en visitas a diferentes centros, entrevistas con miembros del equipo directivo y del equipo técnico. En la investigación cualitativa se pasó la Escala de evaluación de inclusión (Gutiérrez-Ortega, Hernández-Soto \& Jenaro-Rio, 2019).

Las observaciones se realizaron a través de visitas de una duración de entre 60 a 120 minutos a cada centro. Se realizaron un total de 8 visitas. Los datos se registraron en un diario de visita.

Se realizaron entrevistas semi-estructuradas al equipo directivo y al equipo técnico. Las entrevistas incluyeron preguntas en torno a los cuatro grandes temas que se abordarán en la fase cuantitativa, Comunidad, Valores, Políticas y Prácticas. Los datos fueron codificados para lograr una mayor validez de esta investigación.

Finalmente, se pasó una escala para valorar la inclusión en el centro. El instrumento utilizado para la recogida de datos cuantitativos han sido la Escala de Evaluación de la Inclusión (EEI). La escala se ha elaborado a partir del Index para la inclusión, que ha sido adaptado para ser utilizado en centros de atención a personas con discapacidad. El instrumento consta de 24 ítems que se valoran en una escala tipo Likert de 4 puntos, correspondiendo puntuaciones más elevadas a resultados más inclusivos. Los ítems se agrupan en cuatro factores: Comunidad ( 6 ítems), Valores (5 ítems), Políticas (6 ítems) y Prácticas (7 ítems).

El estudio ha sido llevado a cabo en una organización social, situada en el norte de España, con un fuerte sentido de comunidad. Además, dicha organización ha sido elegida por dos motivos, en primer lugar, porque cumple con todas las características de una organización inclusiva y, en segundo lugar, porque a lo largo de los años ha constituido una comunidad a través de la cual ha creado una empresa conjunta, un repertorio compartido y un compromiso mutuo (Wenger, McDermott \& Snyder, 2002). 
Los participantes del estudio, tanto en la investigación cualitativa como cuantitativa, fueron seleccionados de forma intencional para que todos los colectivos del centro tuvieran representación en el estudio. De este modo, en la fase cuantitativa participaron miembros del equipo directivo, equipo técnico, familias y personas usuarias.

\section{ANÁLISIS DE DATOS}

Como se trataba de un estudio de diseño mixto, los datos para este estudio se organizaron siguiendo varios pasos. En primer lugar, se realizaron los análisis cualitativos donde se utilizaron procesos de codificación de las preguntas realizadas en las entrevistas llevadas a cabo a los miembros del equipo directivo y al equipo técnico. Los procesos de codificación han incluido la identificación de conceptos integrados en los datos, la organización de conceptos discretos en categorías, la definición de las propiedades y dimensiones de las categorías (se han recogido un total de cuatro) y su vinculación de acuerdo con sus propiedades y dimensiones a los temas explicativos (Boghan \& Biklen, 1998). Los datos fueron analizados en Atlas Ti 8 por dos investigadores del estudio, en los casos de no concordancia en la asignación de códigos se analizaron de nuevo por los investigadores participantes.

En segundo lugar, se analizaron los datos de la escala de evaluación de la inclusión pasada a 93 participantes se introdujeron en SPSS. Posteriormente, los datos se analizaron utilizando estadísticas descriptivas de frecuencia y porcentajes. Posteriormente, utilizando una tabla de medias y un procedimiento de análisis de varianza (ANOVA), se analizaron las diferencias de medias y, finalmente, se analizaron las diferencias entre los cuatro grupos de participantes para ver si había diferencias significativas en las percepciones. Se usó un valor crítico de 0,05 para determinar la importancia. Si se encontró una diferencia significativa, se utilizó una prueba de comparación múltiple post-hoc para determinar qué grupos diferían significativamente entre sí.

\section{RESULTADOS}

En primer lugar, se presentan los resultados de la parte cualitativa. De las entrevistas personales a miembros del equipo directivo y profesionales surgieron tres temas principales en el análisis. La importancia de la participación (Prácticas), los derechos de las personas (Valores), y la implicación de un proyecto común (Comunidad). 
El análisis de los datos de observación reveló aspectos asociados principalmente a las personas usuarias, lo cual ha ayudado a complementar los datos de las entrevistas realizadas a miembros del equipo directivo y al equipo técnico. Se ha observado que en los mensajes transmitidos por las personas en los diferentes centros siempre subyacen dos temas, el centro como proyecto de vida (Prácticas) y la importancia de valorar a las personas (Valores).

De los resultados de los análisis cuantitativos obtenidos por la organización analizada podemos señalar que han en ningún factor (Comunidad, Valores, Políticas y Prácticas), en una escala de 1 a 4 , se obtienen resultados inferiores a tres.

La Dimensión Cultura Inclusiva ha obtenido una media de 3,61 en el factor Valores y 3,21 en el factor Comunidad; la Dimensión Políticas Inclusivas ha obtenido una puntuación de 3,50 y la Dimensión Prácticas Inclusivas 3,41 de media.

Estos resultados no se ven afectados, aunque varíen las características de los participantes: la edad, el género, el rol que se desempeñe en el centro o el tiempo de relación con él. Por lo que se puede ver analizando resultados de los ítems es que no hay diferencias de opinión entre los diferentes miembros de la comunidad en torno a los temas relacionados con la cultura, las políticas y las prácticas inclusivas.

En los resultados obtenidos destacan las puntuaciones en el factor Cultura Inclusiva ya que es el que mayores puntuaciones ha obtenido, lo cual es coherente tanto con lo analizado en los datos recogidos en las observaciones y en las entrevistas realizadas.

Los resultados obtenidos a través de las diferentes fuentes están vinculados ya que la participación y la importancia de la persona, es un elemento clave en la cultura de la organización, factor mejor puntuado. Como señalaba un miembro del equipo directivo de la organización estudiada "es fundamental creer en las posibilidades de las personas, aumentar sus capacidades de elección y ofrecer apoyos para mantener la individualidad" y este aspecto señala otra persona del equipo técnico "está en nuestro ADN".

A partir de todos los conjuntos de datos podemos decir que cuando hay un fuerte sentimiento de comunidad la transmisión de los valores inclusivos es totalmente trasversal a la organización. Y, que independientemente, de las características individuales de las personas el sentimiento hacia la diversidad y la inclusión es el mismo. La utilización de 
diferentes fuentes de datos nos ha ayudado a realizar la convergencia entre los enfoques cualitativos y cuantitativos ha ocurrido dentro de la investigación de métodos mixtos. Como señala Teddlie y Tashakkori (2009), los resultados de un método nos han ayudado a identificar preguntas para a utilizar por el otro método.

\section{CONCLUSIONES}

El análisis de las características de la comunidad que ha participado en el estudio tanto desde el punto de vista cualitativo como cuantitativo nos permite concluir señalando que las culturas, políticas y prácticas inclusivas, como indican Ainscow, Booth y Dyson (2006), no se producen en el vacío, sino que están inmersas en los procesos de aprendizaje social que se desarrollan en los contextos organizativos. Dichos contextos tienen el poder de influir en las personas que ponen en marcha las prácticas inclusivas y, por tanto, en su forma de pensar (Ainscow \& Sandill, 2010). Por lo que podemos dar una respuesta positiva a la primera pregunta de nuestro estudio, ya que claramente el sentimiento de comunidad ha influido en la existencia de percepciones compartidas hacia la inclusión.

Dando respuesta a la segunda pregunta que nos planteábamos, se puede concluir señalando que es organización inclusiva ya que ha obtenido en todos los aspectos analizados a nivel cuantitativo altas puntuaciones. Aspecto que se ha visto apoyado por los datos extraídos de la investigación cualitativa, ya que tanto en las visitas a los centros realizadas como en las entrevistas que se han codificado en el estudio se puede ver que hay un lenguaje común, una cultura y una visión compartida marcada por la defensa de los derechos de las personas y valoración de las diferencias de las diferentes personas que forman parte de su comunidad. Además de ello, no ha habido diferencias independientemente del rol que la persona ocupara en la comunidad, su edad o su sexo.

Para finalizar, nos planteábamos si una metodología de investigación mixta sería adecuada para analizar un constructo complejo como es el de la inclusión.

Podemos concluir señalando que ha sido muy enriquecedor la realización de una primera fase cualitativa ya que a la hora de analizar los datos cuantitativos derivados de la escala ha sido mucho más fácil entender que todos los colectivos presenten puntuaciones similares en todos los factores analizados. La investigación se ha llevado a cabo como una secuencia natural y ha ayudado a los investigadores a comprender matices que hubieran quedado ocultos de no utilizar un enfoque mixto. 
En definitiva, se puede indicar que los métodos mixtos nos han permitido abordar un constructo complejo desde diferentes perspectivas. $\mathrm{Y}$ aunque la flexibilidad y la experimentación son características importantes del proceso de diseño en un estudio exploratorio cualitativo (Miles \& Huberman, 1994; Creswell, 2009; Teddlie \& Tashakkori, 2009 ), se ha visto en la que el rigor metodológico (es decir, la aplicación de los principios y reglas metodológicas que se establecieron durante el proceso de diseño de la investigación) y un enfoque sistemático son necesarios cuando se realiza un estudio exploratorio para proporcionar la base firme para la posterior integración e interpretación de los datos.

Para finalizar nos gustaría señalar algunas limitaciones del estudio. Como el estudio presentado se ha llevado a cabo en una única entidad, creemos que sería enriquecedor realizar un estudio multicaso que nos permita analizar diferentes comunidades y ver como influyen las características de cada una de ellas en los resultados.

\section{REFERENCIAS}

Ainscow, M., \& Sandill, A. (2010). Developing inclusive education systems: the role of organisational cultures and leadership. International Journal of Inclusive Education, 14(4), 401-416

Amor, A. M., Hagiwara, M., Shogren, K. A., Thompson, J. R., Verdugo, M. Á., Burke, K. M., y Aguayo, V. (2019). International perspectives and trends in research on inclusive education: a systematic review. International Journal of Inclusive Education, 23(12), 1277-1295. https://doi.org/10.1080/13603116.2018.1445304

Azorín, C.M. \& Ainscow, M. (2018). Guiding schools on their journey towards inclusion. International Journal of Inclusive Education,24(1), 58-76. https://doi.org/10.1080/13603116.2018.1450900

Cox, T., \& Cox, T. J. (2001). Creating the Multicultural Organization; A Strategy for Capturing the Power of Diversity. San Francisco: Jossey-Bass.

Creswell J.W., Fetters M.D. \& Ivankova N.V. (2004). Designing a mixed methods study in primary care. Ann Fam Med, 2,7-12.

Creswell, J. W. (2009). Research Design, Qualitative, Quantitative and Mixed Methods. 3rd edn. Sage: Thousand Oaks, CA.

Creswell, J.W. (2014). Research design: qualitative, quantitative, and mixed methods approaches. Thousand Oaks, CA: Sage.

Greene, J. C., \& Caracelli, V. J. (2003). Making paradigmatic sense of Mixed Methods practice. En A. Tashakkori \& C. Teddlie (Eds.), Handbook Mixed Methods in social and behavioral research (pp. 91- 110). Thousand Oaks, CA: Sage.

Gutiérrez-Ortega, M., Hernández-Soto, R. \& Jenaro-Río, C. (2019). Escala de Evaluación de la Inclusión (EEI) para centros y servicios para personas con discapacidad: validación preliminar. Revista de Educación Inclusiva, 12(1), 97-118.

Hiebert, J., Gallimore, R. \& Stigler, J. (2002). A Knowledge Base for the Teaching Profession: What Would It Look Like and How Can We Get One? Educational Researcher, 31, 3-15.

https://doi.org/10.3102/0013189X031005003 
Miles M.B. \& Huberman A.M. (1994). Qualitative Data Analysis. An Expanded Sourcebook. 4nd edn. Sage: Thousand Oaks, CA

Schein, E. H. (2004). Organizational Culture and Leadership. San Francisco: John Wiley and Sons.

Tashakkori, A., \& Teddlie, C. (2003). Handbook of mixed methods in the social and behavioral sciences. Thousand Oaks, CA: Sage.

Teddlie, C. \& Tashakkori, A. (2009). Foundations of Mixed Methods Research. Sage: London.

Wenger, E., McDermott, R. \& Snyder, W. (2002). Cultivating communities of practice: A guide to managing knowledge. Cambridge, MA: Harvard University Press. 